\title{
PLEIADES-HR IMAGE QUALITY COMMISSIONING
}

\author{
Laurent Lebègue, Daniel Greslou, Françoise deLussy, Sébastien Fourest, \\ Gwendoline Blanchet, Christophe Latry, Sophie Lachérade, Jean-Marc Delvit, \\ Philippe Kubik, Cécile Déchoz, Virginie Amberg, Florence Porez-Nadal \\ CNES 18, avenue Edouard Belin, 31401 TOULOUSE CEDEX 4 France \\ Phone: 33.(0)5.61.27.39.97 Fax: 33.(0)5.61.27.31.67 E-mail: laurent.lebegue@cnes.fr
}

ISPRS and IAA : Pléiades Inflight Calibration and Performance Assessment

KEY WORDS: Image Quality, Radiometry, Geometry, Calibration

\section{ABSTRACT:}

PLEIADES is the highest resolution civilian earth observing system ever developed in Europe. This imagery program is conducted by the French National Space Agency, CNES. It operates since 2012 a first satellite PLEIADES-HR launched on 2011 December 17th, a second one should be launched by the end of the year. Each satellite is designed to provide optical $70 \mathrm{~cm}$ resolution coloured images to civilian and defence users. The Image Quality requirements were defined from users studies from the different spatial imaging applications, taking into account the trade-off between on-board technological complexity and ground processing capacity. The assessment of the image quality and the calibration operation have been performed by CNES Image Quality team during the 6 month commissioning phase that followed the satellite launch. These activities cover many topics gathered in two families : radiometric and geometric image quality. The new capabilities offered by PLEIADES-HR agility allowed to imagine new methods of image calibration and performance assessment.

Starting from an overview of the satellite characteristics, this paper presents all the calibration operations that were conducted during the commissioning phase and also gives the main results for every image quality performance.

\section{INTRODUCTION TO PLEIADES-HR SATELLITE}

The PLEIADES program is a space Earth Observation system led by France, under the leadership of the French Space Agency (CNES). It will operate in 2012 two agile satellites designed to provide optical images to civilian and defence users.

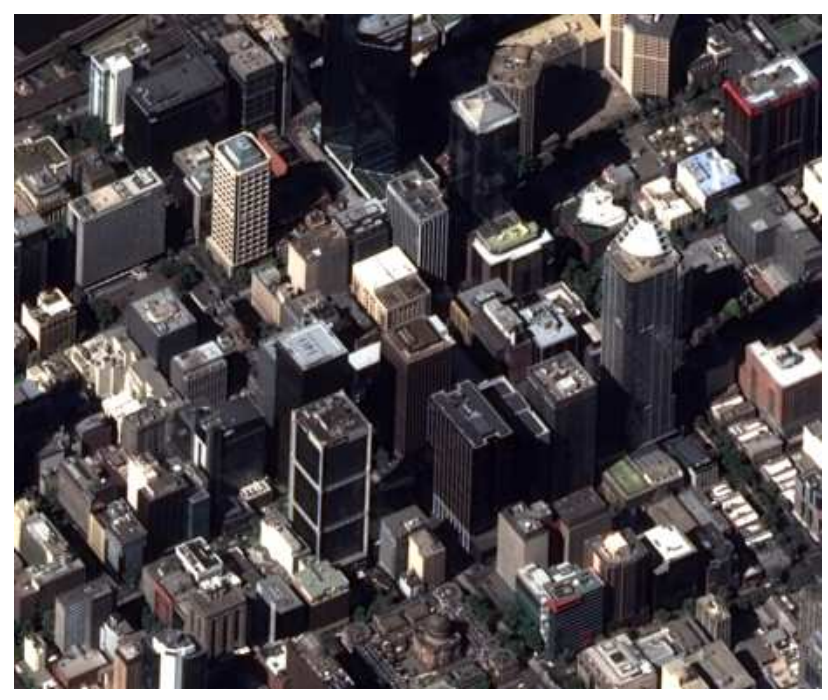

Figure 1 : Pleiades-HR image of Melbourne downtown, (C) CNES Copyright 2012

Since it was successfully launched on December 17th, 2011, Pleiades 1A high resolution optical satellite has been thoroughly tested and validated during the commissioning phase led by CNES.
Images are simultaneously acquired in Panchromatic (PA) and multi-spectral (XS) mode, which allows, in nadir acquisition condition, to deliver $20 \mathrm{~km}$ wide, false or natural coloured scenes with a $70 \mathrm{~cm}$ ground sampling distance after PA+XS fusion. The scan-line is constructed by 5 detector arrays for PA and XS bands with overlapping Inter-Array-Zone (IAZ) to ensure line continuity.

Coverage will be almost world-wide with a revisit interval of $24 \mathrm{~h}$ for 2 satellites when Pleiades 1B will be launched by the end of the year.

The Image Quality requirements were defined thanks to users surveys from the different spatial imaging applications, taking into account the trade-off between on-board technological complexity and ground processing capacity.

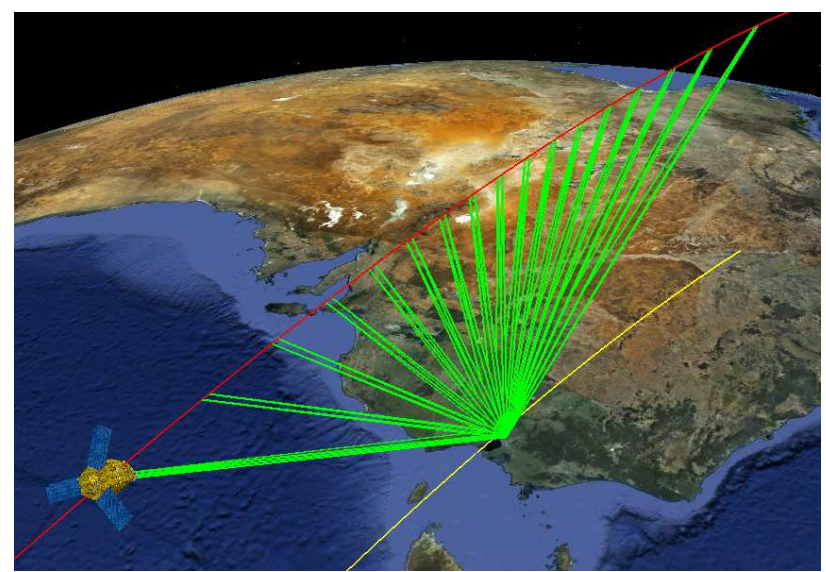

Figure 2 : example of video acquisition mode on Melbourne

The major constraints of weight and agility led to the development of a highly compact satellite (about 1 ton weight), 
to minimize the moments of inertia [14]. Agility is a characteristic which allows the satellite to acquire off-nadir targets rapidly in a large flight envelope, in order to sequence a large number of images. This agility is imposed by several requirements stated by the users. For instance, a 100x100 km2 zone can be acquired by the satellite from the same orbit thanks to a lateral multi-band coverage. As for stereoscopic capacities, 3 images from the same zone can be acquired in a single pass with $\mathrm{B} / \mathrm{H}$ lying between 0.1 and 0.5 . During the commissioning phase, video mode has been realized thanks to more than 30 images of the same target acquired in 5 minutes (Fig 2).

The image quality activities were performed during 6 months at the Pleiades Image Calibration Center (ICC) at CNES. New brand methods have been applied taking advantage of the platform agility.

\section{RADIOMETRIC ACTIVITIES}

\subsection{MTF assessment and refocusing operations}

Refocusing operations have been conducted thanks to 3 methods. The main one uses stars [3] and [8] acquired for different adjustments of the refocusing system made of a thermal control of the telescope's secondary mirror position. It allows to measure by the way a very accurate $2 \mathrm{D}$ assessment of the MTF, (Fig. 3) as the Point Spread Function (PSF) is the image of a point-like source of light as seen by the instrument [5]. Thanks to this very efficient method, the instrument was refocused 5 days after launch.
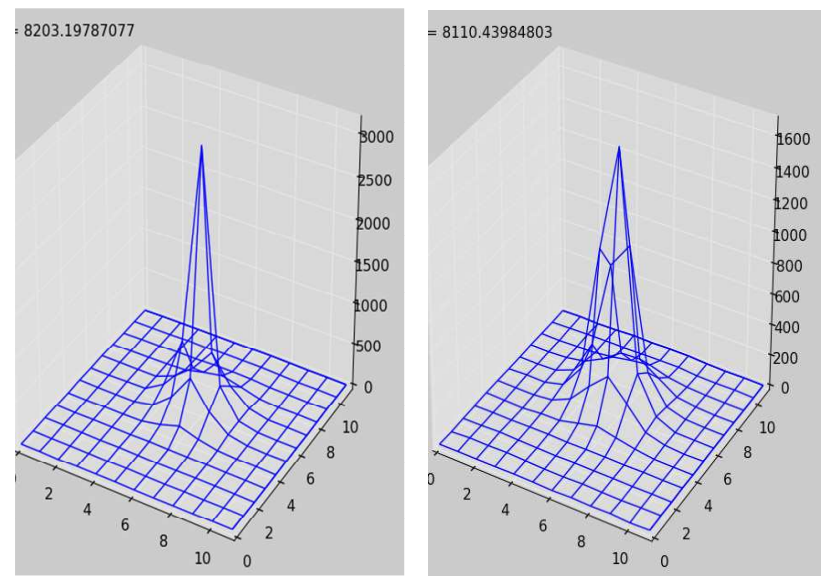

Figure 3 : example of PA MTF measured on stars for different defocusing adjustments

The second one is based on a neural network with monitored defocused terrestrial images as input (Fig. 4). It gave results coherent with the star method.
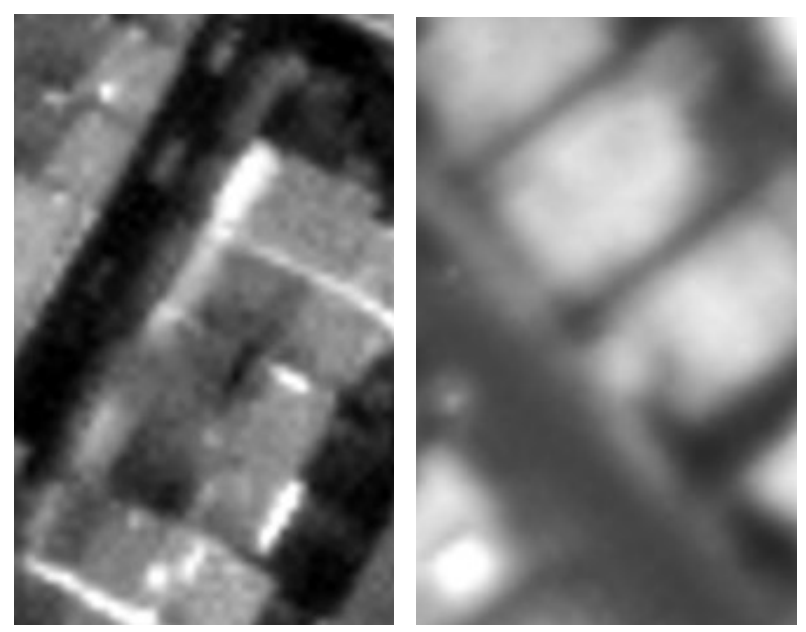

Figure 3 : example of PA defocused images

The third method uses the offset measured between homologous PA pixel of IAZ as this offset is geometrically related to the defocusing. This passive method is used to monitor the evolution of the instrument defocus (Fig. 5)

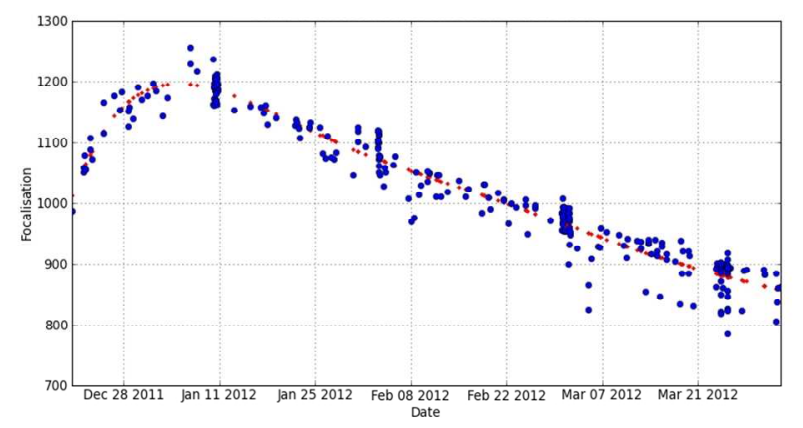

Figure 5 : defocus evolution between launch and April 1,2012

\subsection{Absolute calibration}

Several methods are combined to synthesis the absolute calibration coefficients for every spectral bands [7].

First methods are absolute as they relate Pleiades images digital count to the theoretical signal expected for different kinds of landscape and environmental conditions. For instance, numerous acquisitions over the oceans have been processed using Rayleigh properties for B0, B1 and B2 bands (B3 used to characterize aerosols contribution). The main difficulty was to avoid cloud coverage over oceans. We also used images of La Crau test site equipped with the CIMEL radiometer providing automatic measurement of multi-angular viewing of atmosphere and close neighbouring ground reflectance. Moreover, a video made of 24 acquisitions has been realized to study the measures sensitivity to satellite viewing angles.

Other methods are relative as they compare Pleiades images to another reference. For instance, we inter-calibrate several sensors such as SPOT, MODIS, PARASOL on steady landscape of sand deserts and Antarctic Dome sites.

Finally, we monitor temporal evolution of absolute calibration using Moon images (Fig. 6) as the Earth satellite is a perfect photometric benchmark accessible without cloud constraints and any operational programming conflict. 


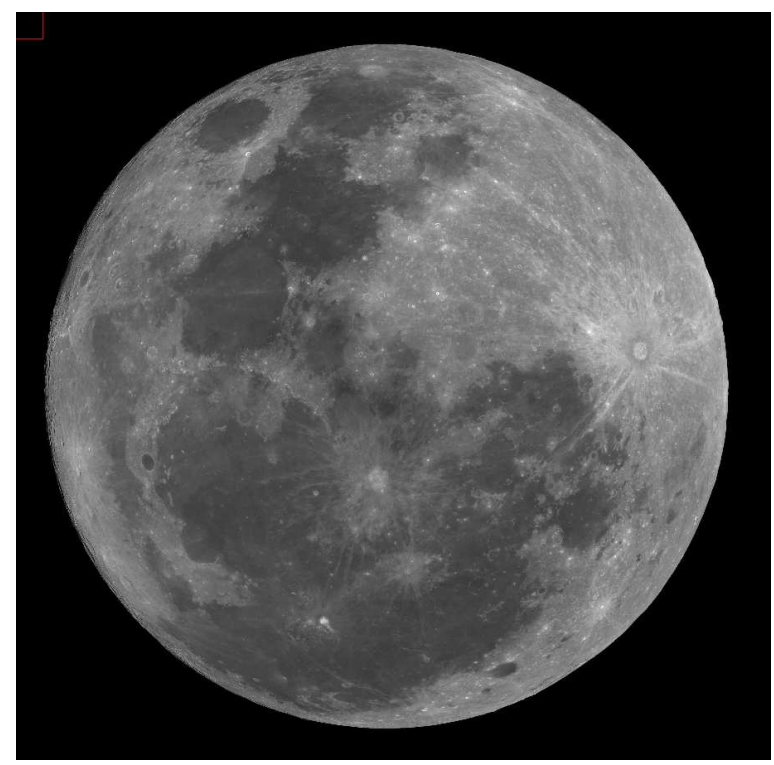

Figure 6 : PA image of the Moon used for absolute calibration

\subsection{Inter-detector normalization}

Because of inter-detector sensitivity differences, the image of a uniform landscape is striped vertically. Detector normalization aims at correcting these relative sensitivities and delivering uniform images of uniform areas. As Pleiades-HR radiometric model is non-linear, the normalization parameters identification requires observation of several uniform landscapes and may be actually very difficult to run, because of the uniformity constraint. This reference method has been operated during the commissioning, but, in order to reduce the in-flight operations, the so-called AMETHIST method [2] has been processed for the first time. An efficient way to bypass the quest of uniformity is to use the satellite agility in order to align the ground projection of the scan-line on the ground velocity. This weird viewing principle (Fig. 7) allows all the detectors to view the same landscape and produce weird raw images as shown in Fig. 8.

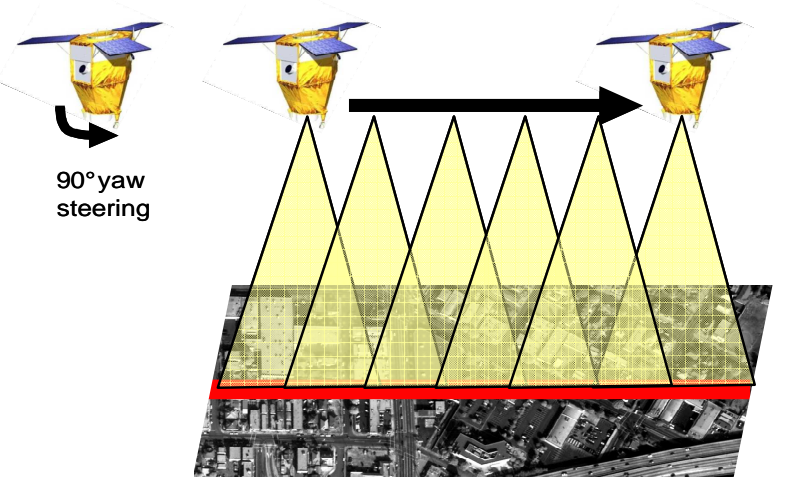

Figure 7 : AMETHIST acquisition principle

After a pre-processing that globally shifts each column of the raw image, we get an image that contains all needed information. This means that every row contains the set of detectors response to the same landscape. Thus, non-linear normalization coefficients can be computed by a histogram matching method.

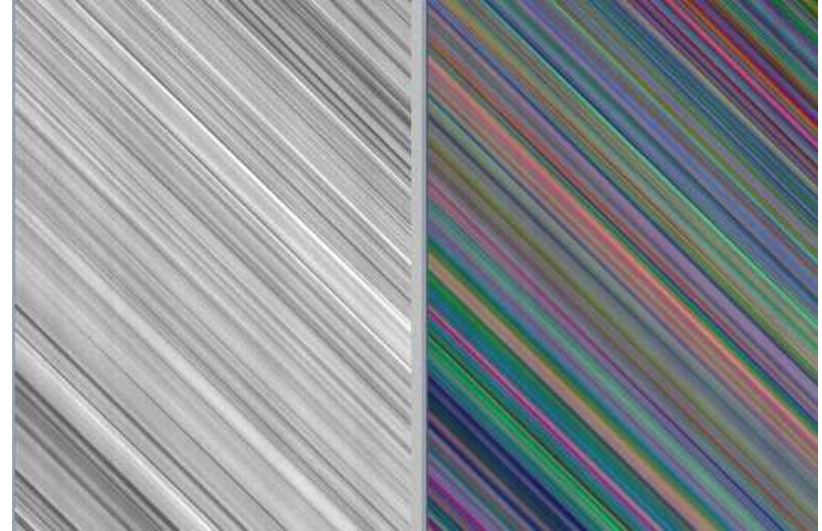

Figure 8 : example of AMETHIST PA and XS images

An AMETHIST campaign [9] requires less than 30 acquisitions on various landscape to fulfill the calibration needs. The coefficients update frequency is expected to be 6 months.

\subsection{Signal-to-noise ratio assessment}

Several brand new techniques were tested to assess the instrumental signal-to-noise ratio. The major one benefits from a dedicated guidance called "slow-motion" [1]. The principle is to steer the satellite so that the projection of the scan-line on the ground remains constant along the image (Fig. 9).

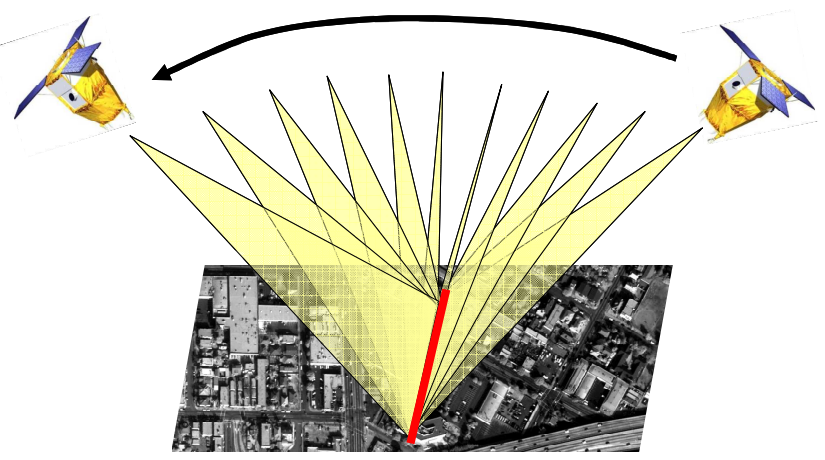

Figure 9 : slow-motion acquisition principle

Therefore, each elementary detector sees the same point on the ground along the column-wise direction with a slight change in the viewing direction. An example of the obtained image is shown in Fig. 10. where it becomes easy to compute the temporal evolution of each elementary detector response for a set of various input radiance. In the end, we can assess the instrumental noise model and its contribution in the radiometric signal to noise ratio budget.

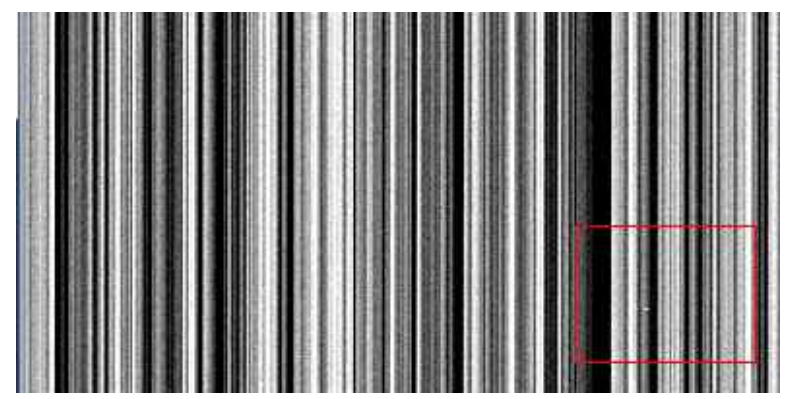

Figure 10 : example of slow-motion image 
Another new technique called BETSI [9] consists in measuring high frequencies residues after low-frequency filtering with the hypothesis that landscape contribution has been cut-off by instrument PSF.

Both methods were successfully processed during commissioning to retrieve ground measurements.

\subsection{Onboard compression bit rate optimization}

The nominal onboard compression bit rate was optimized on numerous images by qualitative and quantitative analysis. Thanks to the wavelet compression scheme, images compressed with the higher bit rate (best quality) can be decompressed on the ground for every available programming bit rates (examples in Fig. 11). Compression artefacts such as sheared contour, wavelet "butterfly" pattern or excessive blur were searched and compared between these images. In the end, the initial recommended bit rates have been increase from 2.50 to 2.86 for PA band and from 2.86 to 3.33 for XS bands.
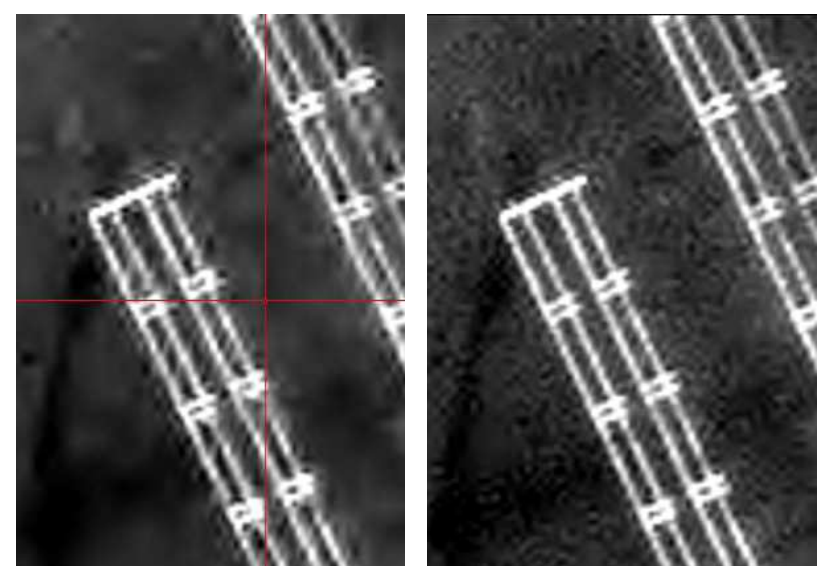

Figure 11 : example of compression artefacts for 2.0 and 4.0 bpp bit rate

\subsection{Restoration and PAN-sharpening}

Given that XS bands MTF is rather high, some aliasing effects are detrimental on images such as irisation in the neighbouring of high radiometric transitions (Fig 12). These defects appear particularly on PAN-sharpened images where XS bands are zoomed by a 4 factor [10]. Ground processing parameters including restoration, re-sampling and interpolation filters have been optimized to reduce these effects. Moreover a new technique of fusion is now being implemented to suppress them.
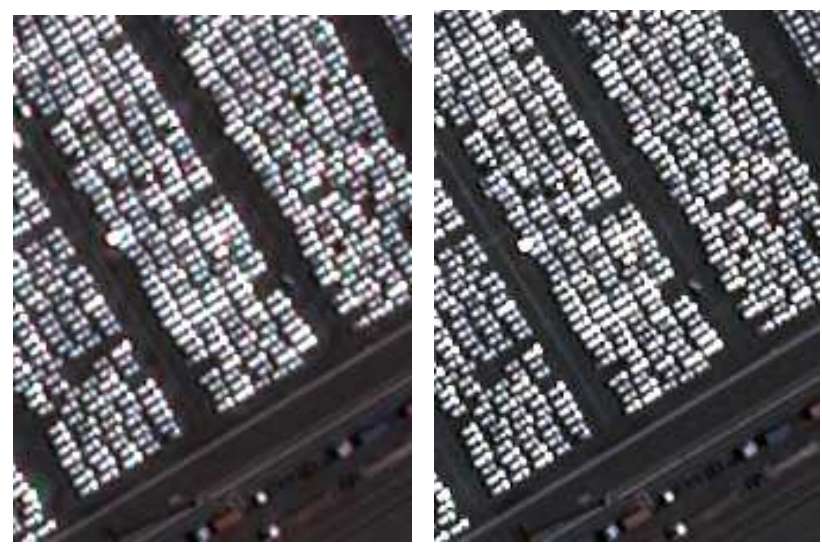

Figure 12 : example of PAN-sharpen images for initial and new techniques

\subsection{Clouds auto-detection}

The algorithm used to detect cloud masks on quick-look images is based on Support Vector Machine (SVM) techniques and is fed by radiometric (mainly bands ratio) and geometric criteria. These criteria weights are tuned during a learning phase. Geometric criteria concerns the parallax measured for very high objects, such as clouds, between the PA and the XS acquisitions as shown in Fig. 13.
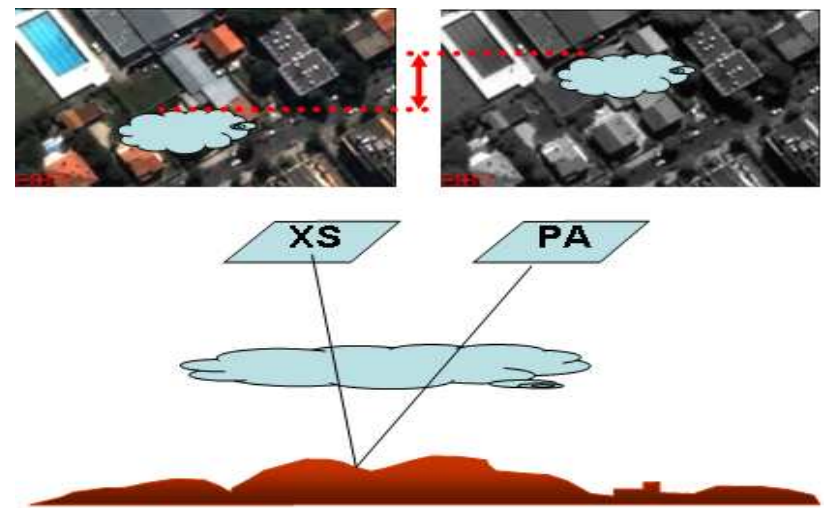

Figure 13 : clouds detection thanks to PA-XS parallax

The learning phase has been conducted on a set of representative landscapes and weather conditions to fulfill the objectives of detection $(90 \%$ of good detection and $0.5 \%$ of false detection).

\section{GEOMETRIC ACTIVITIES}

\subsection{Localization performance}

A fundamental users requirement concerns the image localization without using Ground Control Points (GCP). The assessment of this performance [12] is based on the statistics of the difference between real and image-given localization of very accurate GPS GCP (Fig. 14). CNES uses a large GCP database covering a wide range of latitude in order to observe orbital and seasonal phenomena.

The major contributor is the attitude estimation whose accuracy evolves during the commissioning phase as the calibration operations and the platform in-orbit behaviour knowledge progress.

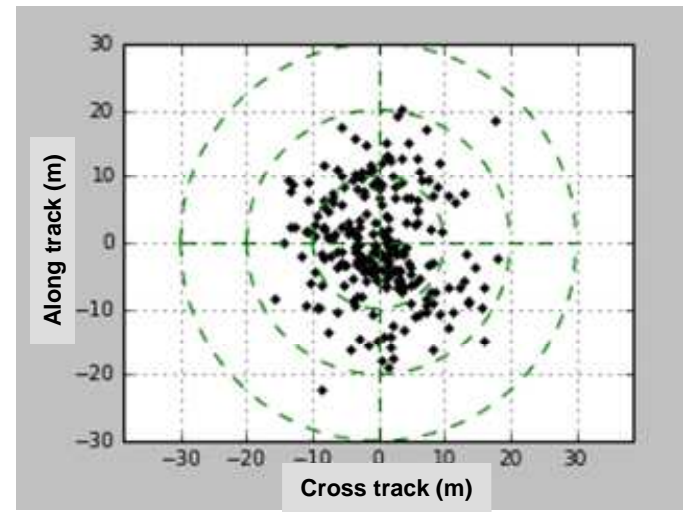

Figure 14 : localization performance measured between launch and April 5, 2012. 
In order to get rid of the use of expensive GCP, a new brand technique called Auto-Reverse [11] has been tested. The idea is to acquire two images of the same site along the same orbit with $180^{\circ}$ angle difference between the azimuth tracks. Hence, correlation of the two images gives roll and pitch double localization biases. The method gives actually very promising results on flat landscapes.

\subsection{Focal plane cartography}

The absolute focal plane cartography, which means the assessment of the accurate viewing directions of every pixel in the instrumental frame is achieved by correlation of PA images with a reference supersite [11] and [12]. This supersite is located at Toulouse (France) and is made of $10 \mathrm{~cm}$ resolution aerial images covering a $20 \times 20 \mathrm{~km}^{2}$ wide area. Unfortunately, winter is not the best season to avoid clouds and to get enough signal in the North hemisphere and the activities planning suffered from lack of data. Nevertheless, the cartography was achieved within 2 months and shows evolutions from ground models raising to 2 PA pixels (Fig 15.)

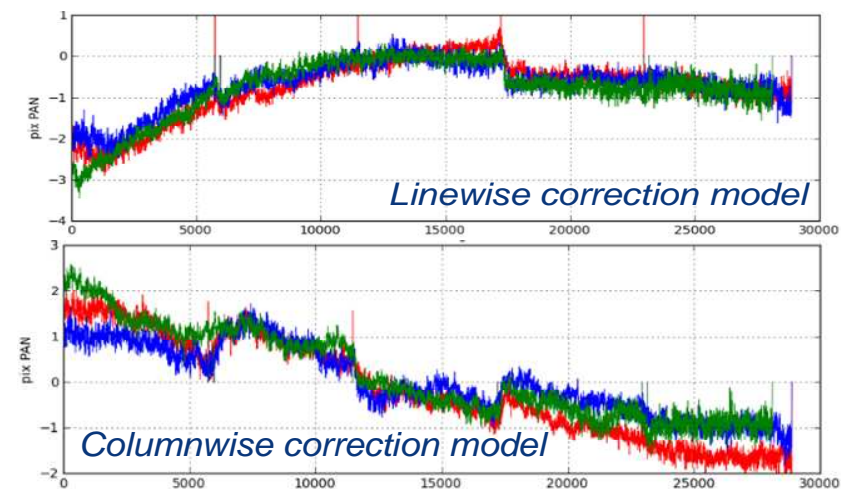

Figure 15 : PA focal plane cartography in-orbit corrections

Concerning, the XS bands, the relative cartography is processed thanks to correlation with the PA band and between XS bands on dedicated flat landscapes. The results accuracy allows to meet the inter-band registration requirements essential for a good PAN-Sharpening.

The commissioning was also the opportunity to test the geometric auto-calibration method [4], [5], and [13] (Fig. 16) which can give cartography without reference site. As for AutoReverse method, first results [11] are very promising when applied on flat landscape.

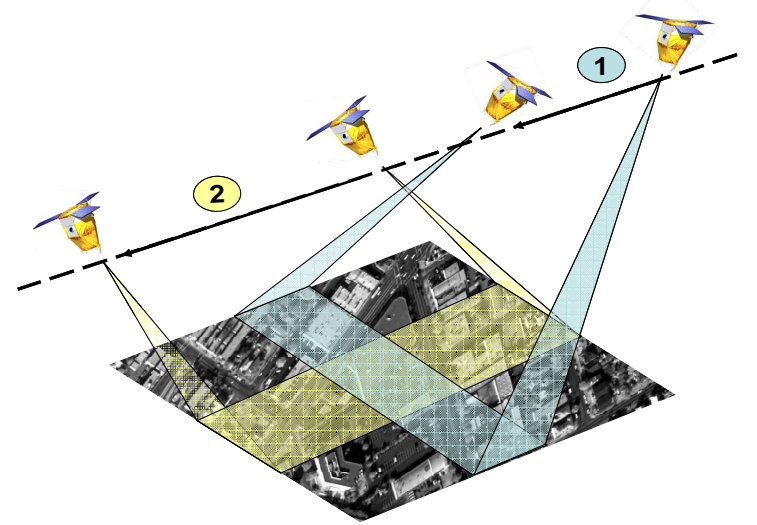

Figure 16 : Geometric auto-calibration acquisition principle

\subsection{Line-of-sight dynamic stability}

Several techniques were used to assess the line-of-sight stability such as inter XS band correlation [4], but the most powerful one uses once again the stars. The idea of our method is to use the stars as references. By definition, a star is stationary in an inertial frame. If the satellite sensor remains pointed at the star [1] and [11], it will create a bright column in the image whose straightness depends on the line-wise behaviour of the potential micro-vibrations. A dedicated guidance, called starAcq, has been designed to fulfill these needs (Fig. 17).

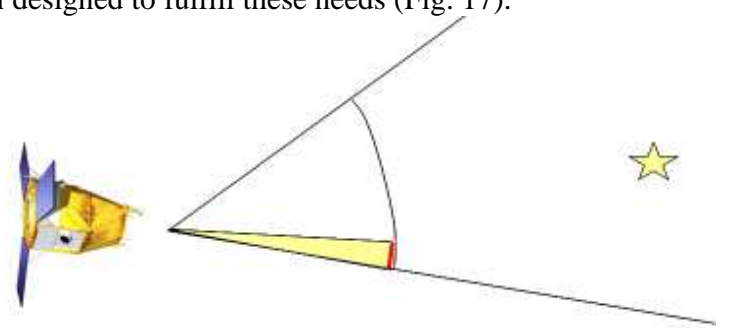

Figure 17 : starAcq guidance principle

Thanks to this method, we were able to confirm 4 days after launch, the presence of micro-vibration with very low magnitude $(<0.1 \mathrm{PA}$ pixel) without any consequence on image quality (Fig. 18).

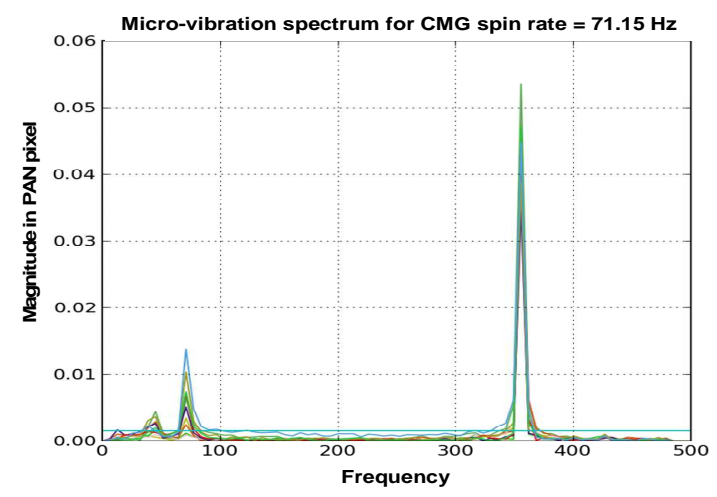

Figure 18 : starAcq method micro-vibrations measurement

As the potential micro-vibration depends on the Gyroscopic Actuators (CMG) spin rate, the method has been applied for different monitoring of this rate in order to optimize agility and image quality (Fig. 19). Fortunately, image quality is preserved for the best agility.

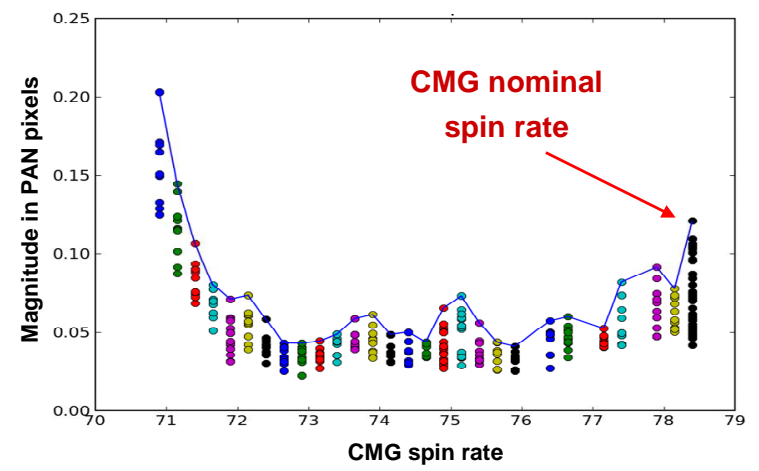

Figure 19 : maximum micro-vibrations magnitude vs CMG spin rate 
Concerning, low and medium frequency perturbations assessment, we correlated phased acquisitions, this means images acquired with a 26-days Pleiades cycle delay, to ensure the reproducibility of the geometric viewing conditions and insensitivity to the relief. Results show magnitude perturbations levels compatible with image quality requirements.

\subsection{D rendering capabilities}

The image quality commissioning was the opportunity to validate high level Pleiades image products such as "Perfect Sensor", "Ortho" and "Mosaic" but also to test Pleiades imagery capability of 3D rendering. In this scope, automatic epipolar geometry re-sampling is applied to stereoscopic pairs in order to produce anaglyphs or DEM generator input data. An example is shown in Fig. 20 on Awacs airplane, using CARMEN DEM generator developed at CNES for a stereoscopic pair with a $\mathrm{B} / \mathrm{H}$ of 0.15 . The altitude accuracy is better than $1.5 \mathrm{~m}$ for these objects.
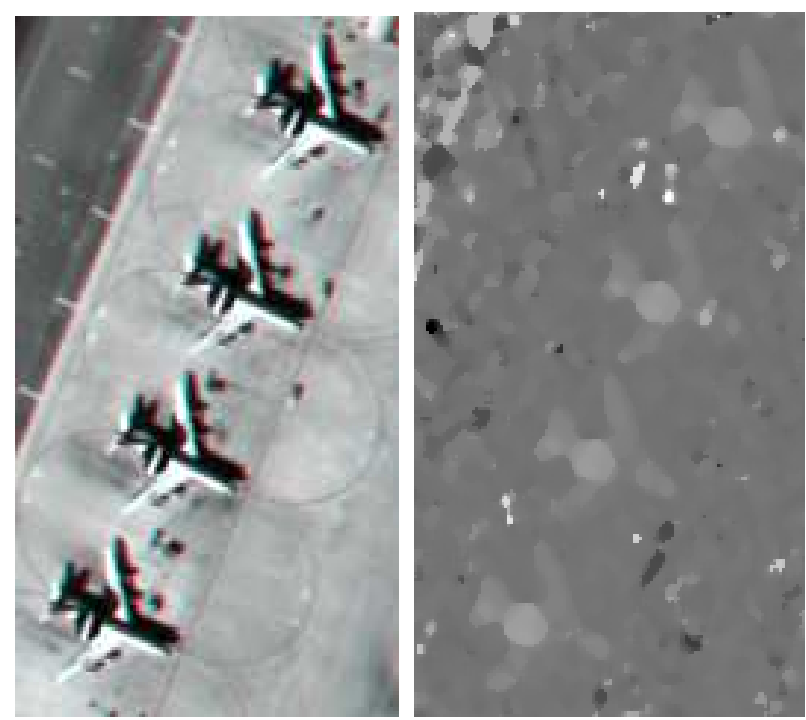

Figure 20 : Awacs airplanes 3D rendering : anaglyph and DEM.

\section{MAIN IMAGE QUALITY PERFORMANCE}

The table below gives the main image quality performance assessed between launch and April 5, 2012. Some of them should be enhanced by the end of the commissioning phase scheduled for June 30, 2012. Concerning localization, the performance has been validated with ground-processing developments available in October 2012. It will then fulfil the $12 \mathrm{~m}$ CE90 requirement. Thus far, the performance is assessed to $16 \mathrm{~m} \mathrm{CE90.}$

\begin{tabular}{|l|c|}
\hline Image Quality criteria & Performance \\
\hline Localization CE90 & $16 \mathrm{~m}$ \\
\hline PAN/XS registration in XS pixels & 0.25 \\
\hline XS/XS registration in XS pixels & 0.15 \\
\hline Planimetry in PAN pixels & 0.20 \\
\hline PAN MTF @ nyquist frequency & 0.16 \\
\hline PAN SNR @ radiance $100 \mathrm{~W} / \mathrm{m}^{2} / \mu \mathrm{m} / \mathrm{sr}-1$ & 150 \\
\hline Absolute calibration accuracy & $5 \%$ \\
\hline
\end{tabular}

Table 1. Main Image Quality performance assessed April 5, 2012.

\section{REFERENCES}

[1] L.Lebègue \& al, "Using Exotic Guidance for PLEIADESHR Image Quality Calibration,” ISPRS Beijing 2008.

[2] Ph.Kubik \& al, "AMETHIST :A MEthod for Equalization Thanks to HISTograms", SPIE Maspalomas 2004.

[3] S. Fourest \& al, "Star-Based Calibration Techniques for PLEIADES-HR Satellites”, CALCON Logan 2009.

[4] JM. Delvit \& al, "Geometric improvement for Earth observation applications", IGARSS Honolulu 2010.

[5] L.Lebègue \& al, "Pleiades-HR Image Quality commissioning foreseen methods", IGARSS Honolulu 2010.

[6] P. Kubik \& al, "First in-flight results of Pleiades 1A innovative methods for optical calibration", ICSO Ajaccio 2012.

[7] S. Lachérade \& al, "Pleiades absolute calibration: first results", ISPRS Melbourne 2012.

[8] S. Fourest \& al, "Star-based methods for Pleiades-HR commissioning”, ISPRS Melbourne 2012.

[9] G. Blanchet \& al, "Pleiades-HR Innovative Techniques for Radiometric Image Quality Commissioning", ISPRS Melbourne 2012.

[10] C. Latry \& al, "Restoration technique for Pleiades-HR panchromatic images", ISPRS Melbourne 2012.

[11] D. Greslou \& al, "Pleiades-HR innovative techniques for Geometric Image Quality Commissioning”, ISPRS Melbourne 2012.

[12] F. deLussy \& al, "Pleiades HR in flight geometrical calibration: Localisation and mapping of the focal plane", ISPRS Melbourne 2012.

[13] JM. Delvit \& al, "Attitude assessment using Pléiades-HR capabilities”, ISPRS Melbourne 2012.

[14] A. Gleyzes \& al, "Pleiades system architecture and main performances”, ISPRS Melbourne 2012.

Reference Web Site for Pleiades Images examples : http://www.flickr.com/photos/pkpro/sets/72157628743311535

\section{ACKNOWLEDGEMENTS}

The author would like to thank the whole team of image experts who have performed a great job, spending time, energy and enthusiasm during the 6 months of the image quality commissioning. He would also like to thank the Pleiades image ground processing team for its support and especially the ICC exploitation and Pleiades Mission teams for their constant good mood, feeding the experts with acquisitions and image products. 